\title{
Translational Motion of a Free Large Polaron and Broadening of Absorption Spectra
}

\author{
Vladimir Mukhomorov \\ Agrophysical Institute, St.-Petersburg, Russia \\ E-mail:vmukhomorov@mail.ru \\ Received February 16, 2010; revised March 17, 2010; accepted March 20, 2010
}

\begin{abstract}
The translational motion of a large polaron as whole is analyzed in the context of its effect on the broadening of an absorption optical spectrum. It was open question how important the role of translational degrees of freedom and the corresponding velocities are on the broadening. The Bogolyubov method of canonical transformation of coordinates is formulated for a system of an electron and field, taking into account rigorous fulfillment of the conservation laws. Separation of variables is carried out for the coordinates describing the translational degrees of freedom and the electron oscillations in a polarization well. The equations obtained for the electronic states explicitly depend on the velocity of the free polaron as a whole. An estimate is made for free polaron in ammonia.
\end{abstract}

Keywords: Large Polaron, Translational Motion, Canonical Transformation, Broadening, Absorption Spectra, Ammonia

\section{Introduction}

ITS is well known that the absorption spectra of free large polaron consists of a broad featureless asymmetric band with a long tail extended to the short-wave length region. There is vast literature on the possible mechanisms of broadening the optical spectrum of free polaron. S. I. Pekar [1] have studied the broadening of the optical absorption spectra of large polaron in crystal as a function of phonon dispersion. In the work [2] authors have calculated the optical absorption coefficient for free polarons using the multiphonons mechanism. The main idea in ref. [3] consist that broadening of the absorption spectra depends on width of an electronic band. In this case broadening of the absorption spectra to proportionally electron effective mass at the bottom of a conductivity band [4]. However the question of a contribution to the broadening from the translational degrees of freedom and from the corresponding velocities is still unanswered. In this work the thermal motion of free quasiparticle as a whole is analyzed in the context of its effect on the broadening of the optical absorption spectra.

\section{Mathematical Method}

To analyze the effect of translational motion of a free large polaron on its absorption spectra, one must separate in the Hamiltonian the translation-invariant degrees of freedom from the coordinates describing the motion of the polaron as whole and derive the velocity dependent equations for the electron transitions. If the electron and quantum field are strongly coupled then the collective localized state of the field and particle is formed. In such a formation the electron motion is rather intricate. On the one hand the electron oscillated within a rather deep polarization potential well and undergoes the optical transitions, and on the other, it moves together with the center of inertia of the system and participates in the translational random walk. The problem is to separate these motions correctly, rigorously taking into account the conservation laws. This can be conveniently done using Bogolyubov [5] method of canonical transformation to the collective coordinates. The Bogolyubov method is the most powerful analytic techniques available for discussing electron-phonon problems. This transformation removes the translational degeneracy and allows one to develop the successive approximation algorithm for the energy and wave function while simultaneously fulfilling the law of conservation of total momentum of the system. Some of the transformed variables are generalized coordinates whose canonically conjugated momenta are the integrals of motion, which are defined by the symmetry properties of the original Hamiltonian and hence ultimately ensure fulfillment of the conservation laws. Fol- 
lowing the Bogolyubov method, we reformulate the adiabatic theory of the particle strongly interacting with the quantum field. The resulting equations determine the electron transitions and depend explicitly on the translational velocity of free polaron.

Within the effective mass one-electron continual approximation the Hamiltonian of the electron-phonon system has the form

$$
\begin{gathered}
H=\frac{p_{r}^{2}}{2 m^{*}}+\gamma^{2} \sum_{f}\left(V_{f}^{(0)} e^{i f r} b_{f}+V_{f}^{(0)^{*}} e^{-i f r}\right)+ \\
\frac{1}{2} \sum_{f} \hbar \omega_{f}\left(b_{f}^{+} b_{f}+b_{f} b_{f}^{+}\right)
\end{gathered}
$$

where the interaction form-factor is defined as $V_{f}^{(0)}=$ $i\left(\hbar \omega_{f} / f u^{1 / 2}\right)(4 \pi / V)^{1 / 2}, u=\left(2 m^{*} \omega_{f} / \hbar\right)^{1 / 2}$ and the dimensionless coupling constant is $\gamma^{2}=\alpha_{c}, \alpha_{c}=\left(1 / \varepsilon_{\infty}\right.$ $\left.-1 / \varepsilon_{s}\right) e^{2} u / 2 \hbar \omega_{f} ; m^{*}$ is the isotropic effective mass of electron, $\omega_{f}$ is the frequency of the long-wave length longitudinal optical phonons of the polar medium; $\varepsilon_{\infty}$ and $\varepsilon_{s}$ are the high-frequency and low-frequency dielectric constants of the isotropically polarizable dielectric continuum, and $\boldsymbol{r}$ is the electron coordinate. The quantum amplitudes $b_{f}$ and $b_{f}^{+}$of the polarization field, respectively, annihilate and create the field quantum $\hbar \omega_{f}$ and obey the Bose-Einstein commutation rule $\left[b_{f}, b_{f^{\prime}}^{+}\right]_{-}=\delta_{f f^{\prime}}$.

In order to develop the iterative procedure for calculating the eigenfunctions and energy eigenvalues of Hamiltonian (1) we modify the canonical transformation of coordinates. For this purpose the electron coordinate is written as the vector sum of two variables

$$
\boldsymbol{r}=\lambda / \gamma+\boldsymbol{q},
$$

where $\boldsymbol{q}$ is independent of $\boldsymbol{r}$ and means the coordinate of the center of gravity of the system, and $\lambda$ describes the electron motion relative to the center. Before developing the perturbation theory, let us introduce in Hamiltonian (1) instead of the phonon creation and annihilation operators, the complex phonon coordinates $q_{f}$ and the corresponding operators of conjugated momentum $p_{f}$ :

$$
q_{f}=\left(b_{f}+b_{-f}^{+}\right) / \gamma \sqrt{2}, \quad p_{f}=i \gamma\left(b_{f}^{+}-b_{-f}\right) / \sqrt{2},
$$

which satisfy the commutation rule $\left[q_{f}, p_{f^{\prime}}\right]_{-}=i \delta_{f f^{\prime}}$. The $1 / \gamma$ factor in (2) and (3) allows one to describe the electron motion relative to the center of inertia even within the lowest-order nonvanishing term of the expansion of the Hamiltonian in $\gamma$ powers. Using Equations (2) and (3) and taking into account that $\partial / \partial \boldsymbol{r}=(\partial \lambda / \partial \boldsymbol{r}) \partial / \partial \lambda$ $=\gamma \partial / \partial \lambda$ one can transform Hamiltonian (1) as

$$
H=\frac{\gamma^{2} \boldsymbol{p}_{\lambda}^{2}}{2 m^{*}}+\gamma^{2} \sqrt{2} \sum_{f} V_{f}^{(0)} q_{f} e^{i f(\boldsymbol{q}+\lambda / \gamma)}+\frac{\gamma^{2}}{2} \sum_{f} \hbar \omega_{f} q_{-f} q_{f}+
$$

$$
\frac{1}{2 \gamma^{2}} \sum_{f} \hbar \omega_{f} p_{-f} p_{f}
$$

One can see that, after changing the variables, the energy of the electron - field interaction and the field potential energy are indeed of the same order in the $\gamma$ parameter. Hamiltonian (4) is translation-invariant. According to Equation (3) the operator of the total momentum of the system can be written as $-i \hbar \partial / \partial \boldsymbol{q}=$ $-i \hbar \partial / \partial \boldsymbol{r}-i \hbar \sum_{f} f q_{f} p_{f}$, so that it is a strict integral of motion. It then follows that the $\boldsymbol{q}$ vector indeed means the coordinate of the center of gravity of the system. Because of a smallness of the last term in Equation (4), the effect of interaction of the electron with the quantum field reduces mainly to the appearance of a potential well [second term in Equation (4)] whose depth depends on the magnitude of the dimensionless coupling constant. As a result of the strong interaction, the quasi-particle is characterized by its own internal structure. The appropriate internal states can be coupled to one another by the electronic transitions.

The interaction of phonons with the charged particles is known to shift the equilibrium positions of the field oscillators relative to their unperturbed values. We thus supplement transformation (2) by the transformation of the field coordinates $q_{f}$ :

$$
q_{f}=\left(u_{f}+Q_{f} / \gamma\right) \exp (-i f r), u_{f}=u_{-f}^{*}, Q_{f}^{+}=Q_{-f}
$$

The translation-invariant $Q_{f}$ variables allow for the quantum fluctuations of the field near its new self-consistent classical value which is determined by the set of $c$-numbers $u_{f}$ to be evaluated in the follows. Within the new variables (5) the interaction potential between the electron and the quantum polarization field retains its order of magnitude in $\gamma$. Note that the introduction of new coordinates (2) results in the appearance of three extra degrees of freedom in comparison to the original system. We therefore impose three additional conditions on the $Q_{f}$ coordinates, which can be chosen in a linear form without loss of generality:

$$
\sum_{f} f v_{f}^{*} Q_{f}=0
$$

This requirements, allow one to retain the number of independent variables after introducing the new electron and field coordinates. The $v_{f}$ values can be chosen in such a way that the orthonormalization condition

$$
\sum_{f} f_{\alpha} f_{\beta} v_{f}^{*} u_{f}=\delta_{\alpha \beta}, \alpha, \beta=1,2,3
$$

is fulfilled together with the requirement that $v_{f}^{*}=v_{-f}$. The coordinate transformations (2) and (5) provide fulfillment of the conservation law for the total momentum. Hamiltonian (4) can be further transformed after the operator of momentum $p_{f}$ is expressed in the terms of the 
new variables $\boldsymbol{q}, \lambda$ and $Q_{f}$ :

$$
p_{f}=-i \frac{\partial}{\partial q_{f}}=-\sum_{f} \frac{\partial Q_{k}}{\partial q_{f}} \frac{\partial}{\partial Q_{k}}-i \frac{\partial \boldsymbol{q}}{\partial q_{f}} \frac{\partial}{\partial \boldsymbol{q}}-i \frac{\partial \lambda}{\partial q_{f}} \frac{\partial}{\partial \lambda} .
$$

Putting $u_{f}$ independent of $q_{k}$ and differentiating (5) with respect to $q_{k}$ we get

$$
\begin{gathered}
\frac{\partial Q_{\boldsymbol{f}}}{\partial q_{\boldsymbol{k}}}=\gamma \frac{\partial q_{\boldsymbol{f}}}{\partial q_{\boldsymbol{k}}} e^{i f \boldsymbol{q}}+i \gamma \boldsymbol{f} q_{\boldsymbol{f}} e^{i f \boldsymbol{q}} \frac{\partial \boldsymbol{q}}{\partial q_{\boldsymbol{k}}}= \\
\gamma e^{i f \boldsymbol{q}_{q}}\left[\delta_{\boldsymbol{f} \boldsymbol{k}}+i \boldsymbol{f}\left(u_{\boldsymbol{f}}+\frac{1}{\gamma} Q_{\boldsymbol{f}}\right) e^{-i f \boldsymbol{q}} \frac{\partial \boldsymbol{q}}{\partial q_{\boldsymbol{k}}}\right] .
\end{gathered}
$$

The derivative $\partial \boldsymbol{q} / \partial q_{\boldsymbol{k}}$ is found by inserting (5) in the additional condition (6) and differentiating the identity obtained. The result is

$$
\sum_{f} f v_{f}^{*}\left(\delta_{k f} e^{i f q}+i f q_{f} e^{i f q} \frac{\partial \boldsymbol{q}}{\partial q_{f}}\right)=0 .
$$

The equation for the partial derivative of $\boldsymbol{q}$ with respect to $q_{k}$ is obtained from Equation (10) taking into account transformation (5) and the condition (7)

$$
\frac{\partial \boldsymbol{q}}{\partial q_{\boldsymbol{k}}}=i \boldsymbol{k} v_{\boldsymbol{k}}^{*} e^{i \boldsymbol{k} \boldsymbol{q}}-\frac{1}{\gamma} \sum_{\boldsymbol{f}}(\boldsymbol{f} \boldsymbol{f}) v_{f}^{*} Q_{f} \frac{\partial \boldsymbol{q}}{\partial q_{\boldsymbol{k}}}
$$

This equation can be solved by iteration, with $1 / \gamma$ as a small parameter. The following solution is then obtained within an accuracy of the terms on the order of $1 / \gamma^{2}$ :

$$
\begin{aligned}
& \frac{\partial \boldsymbol{q}}{\partial q_{\boldsymbol{k}}}=i e^{i \boldsymbol{k} \boldsymbol{q}}\left[\boldsymbol{k} v_{\boldsymbol{k}}^{*}-\frac{1}{\gamma} \sum_{\boldsymbol{f}} \boldsymbol{f}(\boldsymbol{f} \boldsymbol{k}) v_{\boldsymbol{k}}^{*} v_{f}^{*} Q_{\boldsymbol{f}}+\right. \\
& \left.\frac{1}{\gamma^{2}} \sum_{\boldsymbol{f}, l} \boldsymbol{k}(\boldsymbol{f} \boldsymbol{l})(\boldsymbol{f} \boldsymbol{l}) v_{\boldsymbol{k}}^{*} v_{\boldsymbol{f}}^{*} v_{l}^{*} Q_{f} Q_{l}+\ldots\right]
\end{aligned}
$$

Using the transformation of variables (2), one determines the partial derivative $\partial \lambda / \partial q_{f}=-\gamma \partial \boldsymbol{q} / \partial q_{f}$. Now, using this equality and Equation (12), one finally obtains the following expansion series for the $\partial / \partial q_{f}$ operator in terms of the $\lambda, \boldsymbol{q}$, and $Q_{f}$ variables

$$
\begin{gathered}
\frac{\partial}{\partial q_{\boldsymbol{f}}}=e^{i \boldsymbol{q} \boldsymbol{f}}\left\{i \gamma \mathrm{P}_{\boldsymbol{f}}-i \gamma \boldsymbol{f} v_{\boldsymbol{f}}^{*} \frac{\partial}{\partial \lambda}+\sum_{k}(\boldsymbol{f} \boldsymbol{k}) v_{\boldsymbol{f}}^{*} Q_{k} \frac{\partial}{\partial Q_{\boldsymbol{k}}}-\right. \\
\sum_{\boldsymbol{k}} \boldsymbol{k}(\boldsymbol{f} \boldsymbol{k}) v_{\boldsymbol{f}}^{*} v_{\boldsymbol{k}}^{*} Q_{\boldsymbol{k}}\left(i \sum_{l} \boldsymbol{l} u_{\boldsymbol{l}} \frac{\partial}{\partial Q_{l}}-\frac{\partial}{\partial \lambda}\right)+\boldsymbol{f} v_{\boldsymbol{f}}^{*} \frac{\partial}{\partial \boldsymbol{q}}+ \\
\frac{1}{\gamma}\left[\sum_{\boldsymbol{k}, \boldsymbol{l}} \boldsymbol{f}(\boldsymbol{k} \boldsymbol{l})(\boldsymbol{k} \boldsymbol{l}) v_{\boldsymbol{f}}^{*} v_{\boldsymbol{k}}^{*} v_{l}^{*} Q_{\boldsymbol{k}} Q_{l}\left(i \sum_{l} \boldsymbol{l} u_{\boldsymbol{l}} \frac{\partial}{\partial Q_{l}}-\frac{\partial}{\partial \lambda}\right)-\right. \\
\left.\sum_{\boldsymbol{k}} \boldsymbol{k}(\boldsymbol{k} \boldsymbol{f}) v_{\boldsymbol{f}}^{*} v_{\boldsymbol{k}}^{*} Q_{\boldsymbol{k}} \sum_{l} \boldsymbol{l} Q_{\boldsymbol{l}} \frac{\partial}{\partial Q_{l}}-\sum_{\boldsymbol{k}} \boldsymbol{k}(\boldsymbol{k} \boldsymbol{f}) v_{\boldsymbol{f}}^{*} v_{\boldsymbol{k}}^{*} Q_{\boldsymbol{k}} \frac{\partial}{\partial \boldsymbol{q}}\right]+ \\
\frac{1}{\gamma^{2}}\left[\sum_{\boldsymbol{k}, \boldsymbol{l}} \boldsymbol{f}(\boldsymbol{k} \boldsymbol{l})(\boldsymbol{k} \boldsymbol{l}) v_{\boldsymbol{f}}^{*} v_{\boldsymbol{k}}^{*} v_{l}^{*} Q_{\boldsymbol{k}} Q_{l} \sum_{\boldsymbol{m}} \boldsymbol{m} Q_{\boldsymbol{m}} \frac{\partial}{\partial Q_{\boldsymbol{m}}}+\right.
\end{gathered}
$$

$$
\left.\left.\sum_{\boldsymbol{k}, \boldsymbol{l}} \boldsymbol{f}(\boldsymbol{k} \boldsymbol{l})(\boldsymbol{k} \boldsymbol{l}) v_{\boldsymbol{f}}^{*} v_{\boldsymbol{k}}^{*} v_{l}^{*} Q_{\boldsymbol{k}} Q_{\boldsymbol{l}} \frac{\partial}{\partial \boldsymbol{q}}\right]+. .\right\}
$$

where $\mathrm{P}_{f}$ stands for the field generalized momentum; the latter is expressed as a linear combination of the $-i \partial / \partial Q_{f}$ momentum: $i \mathrm{P}_{f}=\partial / \partial Q_{f}-f v_{f}^{*} \sum_{\boldsymbol{k}} \boldsymbol{k} u_{\boldsymbol{k}} \partial / \partial Q_{\boldsymbol{k}}$.

As the $\boldsymbol{q}$ coordinate is a cyclic variable, the corresponding canonically conjugated operator of momentum $-i \hbar \partial / \partial \boldsymbol{q}$ (which coincides with the total momentum of the system) commutes with Hamiltonian (4). Correspondingly, the $\partial / \partial \boldsymbol{q}$ operator will be further replaced throughout by the total momentum $\boldsymbol{P}=\boldsymbol{p}_{r}+$ $\hbar \sum_{\boldsymbol{f}} \boldsymbol{f} b_{\boldsymbol{f}}^{+} b_{\boldsymbol{f}}$. In order to allow for the momentum even in the first approximation, we introduce the $\boldsymbol{I}$ vector such that $\boldsymbol{P}=\gamma^{2} \boldsymbol{I}$. As a result, translational effects appear even in the first order. The total eigenfunction of the system can then be written as

$$
\Psi\left(\boldsymbol{\lambda}, \boldsymbol{q}, Q_{\boldsymbol{f}}\right)=\exp \left(i \gamma^{2} \boldsymbol{I q} / \hbar\right) \Phi\left(\boldsymbol{\lambda}, Q_{\boldsymbol{f}}\right) .
$$

This function realizes a certain representation of the translation group and corresponds to the state with a fixed total momentum $\boldsymbol{P}$ of the system. It is convenient to perform, according to [5], one more unitary transformation of the wave function with respect to the $Q_{f}$ variable and rewrite the total wave function as

$$
\Psi\left(\lambda, \boldsymbol{q}, Q_{\boldsymbol{f}}\right)=\exp \left(i \gamma \sum_{f} s_{f} Q_{\boldsymbol{f}}\right) \exp \left(i \gamma^{2} \boldsymbol{I q} / \hbar\right) \Phi\left(\lambda, Q_{f}\right),
$$

The complex numbers $s_{f}^{*}=s_{-f}$ and can be chosen in a way to satisfy the condition

$$
\sum_{f} f u_{f} s_{f}=0 .
$$

Transformation (15) can be used to expand the collective coordinates Hamiltonian in descending powers of the $\gamma$ parameter:

$$
H=H_{2} \gamma^{2}+H_{1} \gamma+H_{0}+\ldots
$$

where the following notations are used:

$$
\begin{aligned}
& H_{2}=\frac{\boldsymbol{p}_{\lambda}^{2}}{2 m^{*}}+\sqrt{2} \sum_{f} V_{f}^{(0)} u_{f} e^{i f \lambda / \gamma}+\sum_{f} \frac{\hbar \omega_{f}}{2} u_{-f} u_{f}+ \\
& \frac{1}{2} \sum_{\boldsymbol{f}} \hbar \omega_{\boldsymbol{f}}\left(s_{-\boldsymbol{f}}-\frac{i \boldsymbol{I} \boldsymbol{f}}{\hbar} v_{-\boldsymbol{f}}^{*}\right)\left(s_{\boldsymbol{f}}+\frac{i \boldsymbol{I} \boldsymbol{f}}{\hbar} v_{\boldsymbol{f}}^{*}\right) \text {, } \\
& H_{1}=\sum_{f} \hbar \omega_{f}\left(s_{f}+\frac{i \boldsymbol{I} \boldsymbol{f}}{\hbar} v_{f}^{*}\right)\left(\boldsymbol{f} v_{-f}^{*} \frac{\partial}{\partial \lambda}+\mathrm{P}_{-f}\right)+ \\
& \sum_{f}\left[\sqrt{2} V_{f}^{(0)} e^{i f \lambda / \gamma}+\hbar \omega_{f} u_{-f}+\right. \\
& \left.\left(s_{\boldsymbol{f}}+\frac{i \boldsymbol{f I}}{\hbar} v_{f}^{*}\right) \sum_{\boldsymbol{m}} \hbar \omega_{m}\left(s_{\boldsymbol{m}}+\frac{i \boldsymbol{I m}}{\hbar} v_{m}^{*}\right)(\boldsymbol{f m}) v_{-m}^{*}\right] Q_{f}
\end{aligned}
$$




$$
\begin{gathered}
H_{0}=\frac{1}{2} \sum_{f} \hbar \omega_{f} Q_{-f} Q_{f}-\frac{1}{2} \sum_{f} \hbar \omega_{f}\left[2\left(s_{f}+\frac{i \boldsymbol{I} \boldsymbol{f}}{\hbar} v_{f}^{*}\right) \times\right. \\
\left(-\sum_{\boldsymbol{k}} \boldsymbol{k}(\boldsymbol{f} \boldsymbol{k}) v_{-f}^{*} v_{\boldsymbol{k}} Q_{\boldsymbol{k}} \sum_{\boldsymbol{m}} \boldsymbol{m} u_{\boldsymbol{m}} \frac{\partial}{\partial Q_{m}}+\boldsymbol{f} v_{-f}^{*} \sum_{\boldsymbol{k}} Q_{\boldsymbol{k}} \boldsymbol{k} \frac{\partial}{\partial Q_{k}}\right)+ \\
\left(\boldsymbol{f} v_{-f}^{*} \frac{\partial}{\partial \lambda}+\mathrm{P}_{-f}+\boldsymbol{f} v_{-f}^{*} \sum_{\boldsymbol{k}} \boldsymbol{k} s_{\boldsymbol{k}} Q_{\boldsymbol{k}}+i \frac{\boldsymbol{I}}{\hbar} \sum_{\boldsymbol{k}} \boldsymbol{k}(\boldsymbol{f} \boldsymbol{k}) v_{-f}^{*} v_{\boldsymbol{k}}^{*} Q_{\boldsymbol{k}}\right) \\
\left(f v_{f}^{*} \frac{\partial}{\partial \lambda}-\mathrm{P}_{f}+\boldsymbol{f} v_{f}^{*} \sum_{\boldsymbol{k}} \boldsymbol{k} s_{\boldsymbol{k}} Q_{\boldsymbol{k}}+\right. \\
\left.\left.+i \frac{\boldsymbol{I}}{\hbar} \sum_{\boldsymbol{k}} \boldsymbol{k}(\boldsymbol{f} \boldsymbol{k}) v_{f}^{*} v_{\boldsymbol{k}}^{*} Q_{\boldsymbol{k}}\right)\right] .
\end{gathered}
$$

We also require that

$$
\omega_{f}\left(s_{f}+i v_{f}^{*} \boldsymbol{I} f / \hbar\right)=-i u_{f} f \boldsymbol{C} .
$$

The physical meaning of the $\boldsymbol{C}$ vector will be given below. Let us expand the total wave function $\Phi$ and en$\operatorname{ergy} E$ in powers of $\gamma$ :

$$
\begin{aligned}
& E=\gamma^{2} E_{2}+\gamma E_{1}+E_{0}+\ldots, \\
& \Phi=\Phi_{0}+\frac{1}{\gamma} \Phi_{1}+\frac{1}{\gamma^{2}} \Phi_{2}+\ldots
\end{aligned}
$$

Upon substituting (22) in the equation $H \Phi=E \Phi$ with Hamiltonian (17) and collecting the terms with the same $\gamma$ powers, we obtain the following set of equations:

$$
\begin{gathered}
H_{2} \Phi_{0}=E_{2} \Phi_{0}, \\
H_{1} \Phi_{0}+H_{2} \Phi_{1}=E_{1} \Phi_{0}+E_{2} \Phi_{1}, \\
H_{0} \Phi_{2}+H_{1} \Phi_{1}+H_{2} \Phi_{0}=E_{0} \Phi_{2}+E_{1} \Phi_{1}+E_{2} \Phi_{0}, \ldots
\end{gathered}
$$

Because the $\mathrm{H}_{2}$ operator acts only on the field variables $Q_{f}$ the zero-order wave function can be written in a multiplicative form $\Phi_{0}\left(\lambda, Q_{f}\right)=\varphi_{0}(\lambda) \chi\left(Q_{f}\right)$, where $\chi\left(Q_{f}\right)$ is an arbitrary function of the $Q_{f}$ coordinates. Taking into account that the functions $\Phi_{0}$ and $\Phi_{1}$ are ortonormal, one has from the second equation in (23): $\left\langle\varphi_{0}(\lambda)\left|H_{2}-E_{2}\right| \Phi_{1}\right\rangle=0$ so that the $\chi\left(Q_{f}\right)$ function obeys the following equation: $\left\langle\varphi_{0}(\lambda)\left|H_{1}\right| \varphi_{0}(\lambda)\right\rangle \chi\left(Q_{f}\right)$ $=E_{1} \chi\left(Q_{f}\right)$. This equation has a regular solution $\chi\left(Q_{f}\right)$ only if $\left\langle\varphi_{0}(\lambda)\left|H_{1}\right| \varphi_{0}(\lambda)\right\rangle$ is equal to zero, because the $H_{1}$ operator is linear in the $Q_{f}$ variables. Taking into account the form of Hamiltonian (19) and the obvious requirement that $E_{1}=0$, one obtains from (23) the following relation for determining the $u_{f}$ values for an arbitrarily chosen $\chi\left(Q_{f}\right)$ :

$$
\begin{gathered}
\sqrt{2} V_{f}^{(0)}\left\langle\varphi_{0}(\lambda)\left|e^{i f \lambda / \gamma}\right| \varphi_{0}(\lambda)\right\rangle+\hbar \omega_{f} u_{-f}-\left(s_{f}+i \boldsymbol{I} f v_{f}^{*} / \hbar\right) \times \\
\sum_{m} \hbar \omega_{m}\left(s_{\boldsymbol{m}}^{*}-i v_{\boldsymbol{m}} \boldsymbol{m} \boldsymbol{I} / \hbar\right)(\boldsymbol{f} \boldsymbol{m}) v_{\boldsymbol{m}}^{*}=0
\end{gathered}
$$

The Equation (24) is derived under the assumptions that the operators $\mathrm{P}_{f}$ satisfy the condition $\sum_{f} f u_{f} \mathrm{P}_{f}$ $=0$ that directly follows from the $\mathrm{P}_{f}$ definition. Sub- stituting the additional requirement (21) and the condition (7) in (24) and assuming that the ground electronic state is described by the wave function $\varphi_{0}(\lambda)$, one finds from Equation (24) the self-consistent classical field components

$$
u_{f}=-\frac{\sqrt{2} V_{f}^{(0)^{*}} \omega_{f}\left\langle\varphi_{0}(\lambda)|\exp (-i f \lambda / \gamma)| \varphi_{0}(\lambda)\right\rangle}{\hbar\left(\omega_{f}^{2}-(f \boldsymbol{C})^{2}\right)} .
$$

In the strong coupling limit, the $\mathrm{H}_{2}$ term in the Hamiltonian expansion (17) dominates and bears nontrivial information about the system. Using transformations (21) and taking into account that Hamiltonian $\mathrm{H}_{2}$ depends only on the $\lambda$ variable, one can write the energy eigenvalue of the state as $H_{2} \Phi_{0}\left(\lambda, Q_{f}\right)=E_{2} \Phi_{0}\left(\lambda, Q_{f}\right)$, where the notation

$$
E_{2}=W_{2}+\sum_{f} \frac{\hbar}{2} \omega_{f}\left|u_{f}\right|^{2}+\sum_{f} \frac{\hbar}{2} \omega_{f}\left|u_{f}\right|^{2}\left(\frac{f \boldsymbol{C}}{\omega_{f}}\right)^{2}
$$

is introduced. The first two terms in Equation (26) define the internal energy of large polaron.

The equation for determining the lowest energy state wave function $\varphi_{0}(\lambda)$ has the form

$$
\left(\frac{\boldsymbol{p}_{\lambda}^{2}}{2 m^{*}}+\sqrt{2} \sum_{f} V_{f}^{(0)} u_{f} e^{i f \lambda / \gamma}\right) \varphi_{0}(\lambda)=W_{2} \varphi_{0}(\lambda) .
$$

Using Equation (25), it can be recast as

$$
\left(\frac{\boldsymbol{p}_{\lambda}^{2}}{2 m^{*}}-2 \sum_{f} \frac{\omega_{\boldsymbol{f}}\left|V_{\boldsymbol{f}}^{(0)}\right|^{2}\left\langle\varphi_{0}(\lambda)\left|e^{-i f \lambda / \gamma}\right| \varphi_{0}(\lambda)\right\rangle^{i f \lambda / \gamma}}{\hbar\left(\omega_{f}^{2}-(\boldsymbol{f C})^{2}\right)}\right) \varphi_{0}(\lambda)
$$$$
=W_{0} \varphi_{0}(\lambda)
$$

which parametrically depends on the $\boldsymbol{C}$ vector. The integro-differential Equation (28) must be generally solved using the self-consistent method because the classical component of the field is influenced by the electronic state to the same extent as $u_{f}$ influence the electronic state.

Let us now clarify the physical meaning of the $\boldsymbol{C}$ vector. For this purpose, we differentiate $E_{2}$ in (26) with respect to $C$

$$
\begin{array}{r}
\frac{\partial E_{2}}{\partial C_{\alpha}}=\frac{\partial W_{2}}{\partial C_{\alpha}}+\sum_{f} \frac{\hbar \omega_{f}}{2}\left(u_{f} \frac{\partial u_{f}^{*}}{\partial \mathrm{C}_{\alpha}}+u_{f}^{*} \frac{\partial u_{f}}{\partial \mathrm{C}_{\alpha}}\right) \times \\
\left(1+\left(\frac{f \boldsymbol{C}}{\omega_{f}}\right)^{2}\right)+\sum_{f} \hbar\left|u_{f}\right|^{2} f_{\alpha} \frac{(f \boldsymbol{C})^{2}}{\omega_{f}}, \alpha=1,2,3, \quad \text { (29) }
\end{array}
$$

The $\partial W_{2} / \partial \boldsymbol{C}$ derivative can be found using Equation (27). This can be done by differentiating (27) with respect to $C$,

$$
\left(\frac{\boldsymbol{p}_{\lambda}^{2}}{2 m^{*}}+\sqrt{2} \sum_{f} V_{f}^{(0)} u_{f} e^{i f \lambda / \gamma}\right) \frac{\partial \varphi_{0}}{\partial C_{\alpha}}+\sqrt{2} \sum_{f} V_{f}^{(0)} \frac{\partial u_{f}}{\partial C_{\alpha}} e^{i f \lambda / \gamma} \varphi_{0}-
$$




$$
-\frac{\partial W_{2}}{\partial C_{\alpha}} \varphi_{0}-W_{2} \frac{\partial \varphi_{0}}{\partial C_{\alpha}}=0
$$

The average of Equation (30) for the state with the wave function $\varphi_{0}(\lambda)$ is

$$
\frac{\partial W_{2}}{\partial C_{\alpha}}=\sqrt{2} \sum_{f} V_{f}^{(0)}\left\langle\varphi_{0}(\lambda)\left|\frac{\partial u_{f}}{\partial C_{\alpha}} e^{i f \lambda / \gamma}\right| \varphi_{0}(\lambda)\right\rangle
$$

Using the value obtained of the classical field component $u_{f}$ (25), Equation (31) can be transformed to

$$
\sqrt{2} V_{f}^{(0) *}\left\langle\varphi_{0}(\lambda)\left|e^{i f \lambda / \gamma}\right| \varphi_{0}(\lambda)\right\rangle=-\frac{\hbar u_{f}}{\omega_{f}}\left(\omega_{f}^{2}-(f \boldsymbol{C})^{2}\right),
$$

Then, instead of (31), the required derivative can be represented as

$$
\frac{\partial W_{2}}{\partial C_{\alpha}}=-\sum_{f} \frac{\hbar u_{f}^{*}}{\omega_{f}}\left(\omega_{f}^{2}-(f \boldsymbol{C})^{2}\right) \frac{\partial u_{f}}{\partial C_{\alpha}} .
$$

Substituting Equation (33) into (30), we get

$$
\begin{gathered}
\frac{\partial E_{2}}{\partial C_{\alpha}}=\sum_{f} \frac{\hbar(f \boldsymbol{C})^{2}}{\omega_{f}}\left(u_{f}^{*} \frac{\partial u_{f}}{\partial C_{\alpha}}+u_{f} \frac{\partial u_{f}^{*}}{\partial C_{\alpha}}\right)+ \\
\sum_{f} \hbar \omega_{f}\left|u_{f}\right|^{2} f_{\alpha}\left(\frac{f \boldsymbol{C}}{\omega_{f}}\right)^{2}
\end{gathered}
$$

We now determine the $\boldsymbol{I}$ vector. For this purpose, let us multiply condition (21) by $f u_{f}$ and sum over the wave vector $\boldsymbol{f}$. After applying the requirement (7) and condition (16), we obtain the following expression for the $\boldsymbol{I}$ vector:

$$
\boldsymbol{I}=\hbar \sum_{f} \frac{\boldsymbol{f}(\boldsymbol{f C})\left|u_{f}\right|^{2}}{\omega_{f}} .
$$

Let us differentiate (35) with respect to the $\boldsymbol{C}$ vector,

$$
\begin{gathered}
\frac{\partial \boldsymbol{I}}{\partial \boldsymbol{C}}=\hbar \sum_{f} \frac{(\boldsymbol{f} \boldsymbol{f})}{\omega_{f}}\left|u_{\boldsymbol{f}}\right|^{2}+\hbar \sum_{f} \boldsymbol{f} \frac{(\boldsymbol{f C})}{\omega_{f}} u_{f} \frac{\partial u_{f}}{\partial \boldsymbol{C}}+ \\
\hbar \sum_{f} \boldsymbol{f} \frac{(\boldsymbol{f} \boldsymbol{C})}{\omega_{f}} u_{f} \frac{\partial u_{f}^{*}}{\partial \boldsymbol{C}}
\end{gathered}
$$

One can easily see from a comparison of Equation (34) with (36) that

$$
\frac{\partial E_{2}}{\partial C_{\beta}}=\sum_{\alpha} C_{\alpha} \frac{\partial I_{\alpha}}{\partial C_{\beta}}, \alpha, \beta=1,2,3 .
$$

We finally obtain

$$
\begin{aligned}
& \frac{\partial E_{2}}{\partial I_{\alpha}}=\sum_{\gamma} \frac{\partial E_{2}}{\partial C_{\gamma}} \frac{\partial C_{\gamma}}{\partial I_{\alpha}}=\sum_{\beta} C_{\beta}\left(\sum_{\gamma} \frac{\partial I_{\beta}}{\partial C_{\gamma}} \frac{\partial C_{\gamma}}{\partial I_{\alpha}}\right) . \\
& =\sum_{\beta} C_{\beta} \frac{\partial I_{\beta}}{\partial I_{\alpha}}=C_{\alpha}
\end{aligned}
$$

Consequently, the following result is obtained after using the definition for the total momentum $\boldsymbol{P}=\gamma^{2} \boldsymbol{I}$ : $\boldsymbol{C}=\gamma^{2} \partial E_{2} / \partial \boldsymbol{P}$. However, by definition, $\partial E_{2} / \partial \boldsymbol{P}$ is merely the velocity $\mathbf{v}$. Therefore, the $\boldsymbol{C}$ vector is related to the translational velocity of the polaron by expression:

$$
\boldsymbol{C}=\gamma^{2} \partial E_{2} / \partial \boldsymbol{P}=\gamma^{2} \mathbf{v},
$$

and determines to the $\gamma^{2}$ factor, the mean velocity of the center of inertia of system. Hence the energy eigenvalue (28) of the self-consistent ground electronic state $W_{2}$ explicitly depends on the translational velocity of the quasiparticle.

Let us now determine the translational effective mass of the polaron. Using (26) and assuming that the velocity of the center of inertia is small, which ordinary holds for thermal motion, we expand the energy eigenvalue of the system in series

$$
E_{2}^{(0)}=W_{2}^{(0)}+\sum_{f} \frac{\hbar \omega_{f}}{2}\left|u_{f}^{(0)}\right|^{2}+\sum_{f} \frac{\hbar}{2}\left|u_{f}^{(0)}\right|^{2} \frac{(f \boldsymbol{C})^{2}}{\omega_{f}}+\ldots
$$

The quantities that correspond to the zero translational velocity of the polaron are labeled by superscripts in Formula (40). After substituting relation (39) in (40), the following expression is finally obtained for the ground state energy of the system:

$$
E_{2}^{(0)}=W_{2}^{(0)}+\sum_{f} \frac{\hbar \omega_{f}}{2}\left|u_{f}^{(0)}\right|^{2}+\sum_{f} \frac{\gamma^{4} \hbar}{2}\left|u_{f}^{(0)}\right|^{2} \frac{(\boldsymbol{f} \mathbf{v})^{2}}{\omega_{f}}+\ldots
$$

The last term in (41) can be regarded as the kinetic energy of the translational motion of the free particle as a whole: $E_{k i n}=m^{* *} \mathrm{v}^{2} / 2$, where the notation $m^{* *}$ stands for the ground state translational mass of the large polaron:

$$
\begin{gathered}
m^{* *}=\frac{\gamma^{4}}{3} \sum_{f} \frac{\hbar(\boldsymbol{f f})\left|u_{f}^{(0)}\right|^{2}}{\omega_{f}}= \\
\frac{\gamma^{4}}{3} \sum_{f} \frac{2\left|V_{f}^{(0)}\right|^{2}\left|\left\langle\varphi_{0}(\lambda)|\exp (i \lambda f / \gamma)| \varphi_{0}(\lambda)\right\rangle\right|^{2}(\boldsymbol{f f})}{\hbar \omega_{f}^{3}}
\end{gathered}
$$

If the electron is trapped by the polarization field, the interaction of the particle with the field fully "consumes" the rest mass of an electron. Indeed, it follows from the order-of-magnitude analysis of the variables in Equation (42) that the effective mass $m^{* *} \approx \gamma^{8} m^{*}>>m^{*}$ is dominated by the field inertia.

The translational mass $m^{* *}$ can be calculated using (42) if the wave function $\varphi_{0}(\lambda)$ is known. It can be found by solving the nonlinear integro-differential Equation (27). However, in practice, it is convenient to determine the ground state wave function using a direct variational method and varying the total energy functional

$$
F\left[\varphi_{0}(\lambda)\right]=-\frac{\hbar^{2}}{2 m^{*}}\left\langle\varphi_{0}(\lambda)\left|\nabla^{2}\right| \varphi_{0}(\lambda)\right\rangle-\frac{1}{2} \sum_{f} \hbar \omega_{f}\left|u_{f}^{(0)}\right|^{2}
$$


with

$$
u_{f}^{(0)}=-\frac{\sqrt{2} V_{f}^{(0)^{*}}\left\langle\varphi_{0}(\lambda)|\exp (-i f \lambda / \gamma)| \varphi_{0}(\lambda)\right\rangle}{\hbar \omega_{f}} .
$$

The approximate analytic form of a trial variational wave function $\varphi_{0}(\lambda)$ of a nondegenerate ground state can be established by expanding the exponential in Equation (27). Upon restricting ourselves to the quadratic terms in the resultant series, we obtain the oscillator equation

$$
\begin{aligned}
\left\{\frac{\boldsymbol{p}_{\lambda}^{2}}{2 m^{*}}+\sqrt{2} \sum_{\boldsymbol{f}} V_{\boldsymbol{f}}^{(0)} u_{\boldsymbol{f}}^{(0)}\right. & {\left.\left[1+i \frac{\boldsymbol{f} \lambda}{\gamma}-\frac{1}{2}\left(\frac{\boldsymbol{f} \lambda}{\gamma}\right)^{2}+\ldots\right]\right\} \varphi_{0}(\lambda)=} \\
& =W_{2}^{(0)} \varphi_{0}(\lambda)
\end{aligned}
$$

whose solutions are the Hermite polynomials. These functions can be regarded as good approximations to the wave functions of the ground and low excited states of a system with large $\gamma$. Therefore, for slow translational motion of particle we choose the trial ground state wave function in the following analytic form: $\varphi_{0}(\lambda)=\left(\pi^{3}\right.$ $\left.\alpha^{6} \gamma^{6}\right)^{-1 / 4} \exp (-\lambda / \sqrt{2} \alpha \gamma)^{2}$, where $\alpha$ is the variational parameter. Such an approximation for the trial function is consistent with the results of the shifted $-1 / N-$ expansion numerical technique that was applied in [6] and technique solving of nonlinear integral Equation [7] to the analysis of Equation (43).

It was established earlier that the optical transition from the ground to the lowest lying electronic $p$-state is most probable (oscillator strength of 0.77 [1]). Because the transition time $\tau_{0}=(\Delta E / \hbar)^{-1} \approx 10^{-15} s$ is much shorter than the relaxation time $\tau \approx 10^{-13} s$ of the polar lattice, the optical transition can be considered as vertical, i.e., proceeding at a fixed value $u_{f}^{(0)}$ of the classical component of the polarization field in the lowest electronic state. According to this premise and based on Equation (28), the initial electronic state is described by the equation:

$$
\begin{gathered}
\left\{\frac{\boldsymbol{p}_{\lambda}^{2}}{2 m^{*}}-2 \sum_{f} \frac{\left|V_{f}^{(0)}\right|^{2}}{\hbar \omega_{f}}\left\langle\varphi_{0}(\lambda)\left|e^{-i f \lambda / \gamma}\right| \varphi_{0}(\lambda)\right\rangle e^{i f \lambda / \gamma}\right\} \varphi_{0}(\lambda)- \\
2 \sum_{f} \frac{\left|V_{f}^{(0)}\right|^{2}}{\hbar \omega_{f}}\left\langle\varphi_{0}(\lambda)\left|e^{-i f \lambda / \gamma}\right| \varphi_{0}(\lambda)\right\rangle e^{i f \lambda / \gamma}\left(\frac{f C}{\omega_{f}}\right)^{2} \varphi_{0}(\lambda)= \\
\left(W_{0}^{(0)}+W_{0}^{(1)}\right) \varphi_{0}(\lambda),
\end{gathered}
$$

whereas the final state $(k)$ of electronic optical transition obeys the equation

$$
\begin{aligned}
& \left\{\frac{\boldsymbol{p}_{\lambda}^{2}}{2 m^{*}}-2 \sum_{f} \frac{\left|V_{f}^{(0)}\right|^{2}}{\hbar \omega_{f}}\left\langle\varphi_{0}(\lambda)\left|e^{-i f \lambda / \gamma}\right| \varphi_{0}(\lambda)\right\rangle e^{i f \lambda / \gamma}\right\} \varphi_{k}(\lambda)- \\
& 2 \sum_{f} \frac{\left|V_{f}^{(0)}\right|^{2}}{\hbar \omega_{f}}\left\langle\varphi_{0}(\lambda)\left|e^{-i f \lambda / \gamma}\right| \varphi_{0}(\lambda)\right\rangle e^{i f \lambda / \gamma}\left(\frac{f C}{\omega_{f}}\right)^{2} \varphi_{k}(\lambda)=
\end{aligned}
$$

$$
\left(W_{k}^{(0)}+W_{k}^{(1)}\right) \varphi_{k}(\lambda) .
$$

In Equations (45) and (46), the translational velocity of large polaron is assumed to be small, i.e., $(\boldsymbol{f} \mathbf{v})^{2}<\omega_{f}^{2}$ and only quadratic terms are retained in the expansion of the potential. The wave function of the excited electronic $p$-state is chosen in the form

$$
\varphi_{k}(\lambda)=\left(\frac{2}{\pi^{3 / 2} \gamma^{5} \beta^{5}}\right)^{1 / 2} \frac{\lambda}{\gamma} \cos \theta e^{-(\lambda / \sqrt{2} \beta \gamma)^{2}}
$$

where $\beta$ is the variational parameter.

It is convenient to transfer from Equation (45) to the equations

$$
\begin{aligned}
W_{0}^{(0)}+W_{0}^{(1)}= & \left\langle\varphi_{0}(\lambda)\left|\frac{\boldsymbol{p}_{\lambda}^{2}}{2 m^{*}}\right| \varphi_{0}(\lambda)\right\rangle-2 \gamma^{2} \sum_{\boldsymbol{f}} \frac{\left|V_{\boldsymbol{f}}^{(0)}\right|^{2} \rho_{\boldsymbol{f}}^{(0)} \rho_{\boldsymbol{f}}^{(0)^{*}}}{\hbar \omega_{f}}- \\
& 2 \gamma^{2} \sum_{\boldsymbol{f}} \frac{\left|V_{\boldsymbol{f}}^{(0)}\right|^{2} \rho_{f}^{(0)} \rho_{f}^{(0)^{*}}}{\hbar \omega_{f}}\left(\frac{\boldsymbol{f} \mathrm{v}}{\omega_{f}}\right)^{2}, \\
W_{k}^{(0)}+W_{k}^{(1)}= & \left\langle\varphi_{k}(\lambda)\left|\frac{\boldsymbol{p}_{\lambda}^{2}}{2 m^{*}}\right| \varphi_{k}(\lambda)\right\rangle-2 \gamma^{2} \sum_{f} \frac{\left|V_{f}^{(0)}\right|^{2} \rho_{f}^{(0)} \rho_{f}^{(\mathrm{k})^{*}}}{\hbar \omega_{f}}- \\
& 2 \gamma^{2} \sum_{f} \frac{\left|V_{f}^{(0)}\right|^{2} \rho_{f}^{(0)} \rho_{f}^{(\mathrm{k})^{*}}}{\hbar \omega_{f}}\left(\frac{\boldsymbol{f} \mathrm{v}}{\omega_{f}}\right)^{2}
\end{aligned}
$$

Here $\rho_{f}^{(0)}$ and $\rho_{f}^{(\mathrm{k})}$ are the Fourier transforms of electron densities in the ground and excited electronic states. In the adopted approximation, the frequency of the most active electronic dipole transition can be written as

$$
\hbar \Omega_{0 k}=\left(W_{k}^{(0)}-W_{0}^{(0)}\right)+\left(W_{k}^{(1)}-W_{0}^{(1)}\right)=\hbar \Omega_{0 k}^{(0)}+\hbar \Omega_{0 k}^{(1)},
$$

where the second term depends on the polaron velocity, whereas the first term determines the optical transition frequency at the band maximum in the state with the zero center-of-mass velocity. The frequency $\hbar \Omega_{0 k}^{(1)}$ can be found from Equation (48)

$$
\hbar \Omega_{0 k}^{(1)}=S_{0 k} \mathrm{v}^{2} \text {. }
$$

The following notation is used in Equation (50)

$$
S_{0 k}=2 \gamma^{4} \sum_{\boldsymbol{f}} \frac{(\boldsymbol{f} \boldsymbol{f}) \mid \mathrm{V}_{\boldsymbol{f}}^{(0)}}{\hbar \omega_{\boldsymbol{f}}^{3}} \rho_{\boldsymbol{f}}^{(0)}\left(\rho_{\boldsymbol{f}}^{(0)}-\rho_{\boldsymbol{f}}^{(\mathrm{k})}\right) .
$$

Such an approach in the phototransition calculation is justified if the impurity absorption spectrum lies between the IR-absorption region of the polar lattice oscillating and the absorption region of the strongly bound electrons of base material. We assume that the polarons are in thermal equilibrium and that the quasiparticle distribution over the velocities $\mathbf{v}$ is Maxwellian: $F(\mathrm{v})=\pi^{-3 / 2}$ $\mathrm{v}_{0}^{-3} e^{-\left(\mathrm{v} / \mathrm{v}_{0}\right)^{2}}$, where $\mathrm{v}_{0}^{2}=2 k T / m^{* *}$. Then, the full width at half maximum of the optical absorption spectra is related to the standard deviation $D$ as $W_{1 / 2}=2 D \sqrt{2 \ln 2}$. In 
this approximation, the intensity is symmetrically distributed relative to the $\hbar \Omega_{0}^{(0)}$ frequency. The band becomes asymmetric in the presence of photo transitions to high-lying electron excited states. With the Maxwellian velocity distribution, the variance is $D^{2}=\left\langle\Omega_{0 k}^{2}\right\rangle-$ $\left\langle\Omega_{0 k}\right\rangle^{2}=6 S_{0 k}^{2}\left(k T / m^{* *}\right)^{2}$. Then, the band width $W_{1 / 2}$ is equal

$$
W_{1 / 2}=4\left|S_{0 k}\right|\left(k T / m^{* *}\right) \sqrt{3 \ln 2} .
$$

The approach presented to estimating the broadening of the absorption spectra is valid if the inequality $t>c / \Omega_{0 k}^{(0)} \mathrm{v}$ is fulfilled, where $t$ is the mean free path time of the quasi-particle, and $c$ is the light velocity. This inequality is fulfilled for the transition frequencies and temperatures of interest.

\section{Discussions and Conclusions}

The theory is applied to free polaron in ammonia. The electron is self-trapped owing to strong interaction with the quantum polarization field, which is generated by the dipole ammonia molecules librating around their equilibrium positions. Various investigations [8-12] have shown that many properties of electrons in ammonia may be described using the model of continual polarons. Within the framework of this model the possibility of existence coupled of two-electronic bipolaron formations in singlet state $[13,14]$ has been established and magnetic and optical properties of metal-ammonia systems are explained $[8,9]$. The criteria for validity of the theory reduce to the following inequality: $\hbar \omega_{f}<\hbar \omega_{e}<\hbar \omega_{m}$. For an electron in ammonia, $\hbar \omega_{e}=0.885 \mathrm{eV}$ is the energy of the most active optical transition of a self-trapped electron [11], $\hbar \omega_{f} \approx 0.4 \mathrm{eV}$ is the energy of the longitudinal polarization oscillations of the medium [15], and $\hbar \omega_{m}$ $\approx 6 \mathrm{eV}$ is the excitation energy of electrons of the main substance [11]. The orientational oscillations of molecules about their equilibrium position in a polar liquid form elastic waves that may be treated as in crystal. As a result of the directionality and saturation of the intermolecular hydrogen bonds for ammonia, the "quasicrystallinity" of the structure is comparatively well defined. Far from the critical point, the thermal vibrations of the molecules may be reduced to a set of Debye waves, as in a polar crystal, where the spectrum of collective oscillations in the liquid has a cutoff at longer wavelengths than in crystals [16] on account of the translational motion of the particles. The elastic continuum approximation does not generally allow for anisotropy and is far better applicable to a liquid than to a crystal [17].

The width $W_{1 / 2}$ of the optical spectrum of free polaron in ammonia can be numerically estimated if the numerical parameters of the theory are given. At low concentrations of the polarons, the dielectric constants $\varepsilon_{\infty}$ and $\varepsilon_{\mathrm{s}}$ can be set equal to their values in pure ammonia; i.e., $\varepsilon_{\infty}$ $=1.756$ and $\varepsilon_{\mathrm{s}}=22.7$. The electron effective mass $m^{*}$ is usually determined from a comparison of the experimental and theoretical positions of the absorption band maximum. At sufficiently low temperatures, the transition frequency is dominated by the first term in (49). Indeed, for the experimental measurements at temperature $T=225 \mathrm{~K}$ [11], we have the ratio

$$
\frac{\hbar \Omega_{0 k}^{(1)}}{\hbar \Omega_{0 k}^{(0)}}=\frac{S_{0 k} \mathrm{v}^{2}}{\hbar \Omega_{0 k}^{(0)}}=\frac{3 S_{0 k} k T}{m^{* *} \hbar \Omega_{0 k}^{(0)}} \approx \frac{1}{\gamma^{4}}<<1
$$

In this estimate, it is taken into account that, according to Formula (42), the effective mass of the solvated electron is $m^{* *}=0.02 \gamma^{8} m^{*}$. Therefore, the translational velocity contributes only insignificantly to the optical transition. It is mainly determined by the $\hbar \Omega_{0 k}^{(0)}=$ $W_{0}^{(0)}-W_{k}^{(0)}$ term. A comparison of the theoretical position of the band maximum with its experimental value $0.88 \mathrm{eV}[11,18]$ yields the value of $\mathrm{m}^{*}=1.73 \mathrm{~m}$ for the electron effective mass, where $m$ is the mass of a free electron.

Let us estimate numerically the contribution from the translations of quasiparticle as whole to the full width at half maximum of the absorption spectra. For definiteness, we use the following parameter values: $\omega_{0}=5.8 \times$ $10^{13} s^{-1}$ [8], $\gamma^{2}=13.5$. Then, Formulas (51) and (52) yield the value of $W_{1 / 2}=0.23 \mathrm{eV}$ for the contribution from the thermal motion of the quasiparticle, which represents an appreciable part of the experimentally observed value $0.46 \mathrm{eV}[11,18]$. The remaining part in the broadening of the absorption spectra of the polaron is likely to be due to the fluctuations of the polarization field [4] or other mechanisms which short discussed in Introduction. Equations (51) and (52) can be also used to calculate the temperature band-width coefficient; it occurred to be equal to $d W_{1 / 2} / d T=1.03 \times 10^{-3} \mathrm{eV} / \mathrm{K}$. The experimentally measured $[19,20]$ range $(0.6-1.6) \times$ $10^{-3} \mathrm{eV} / \mathrm{K}$ of the temperature coefficient is in satisfactory agreement with the calculated value.

\section{References}

[1] S. I. Pekar, "Research in Electron Theory of Crystals," US AEC Report, 1960.

[2] M. J. Goovaerts, J. M. De Sitter and J. T. Devreese, "Numerical Study of Two-Phonon Sidebands in the Optical Absorption of Free Polarons in the Strong-Coupling Limit," Physical Review B, Vol. 7, No. 6, 1973, pp. 2639-2644.

[3] D. Emin, "Optical Properties of Large and Small Polarons," Physical Review B, Vol. 48, 1993, pp. 1369113696.

[4] Y. T. Mazurenko and V. K. Mukhomorov, "On the Mechanism of Optical Spectra Broadening of Polarons," Optics and Spectroscopy (USSR), Vol. 41, 1976, pp. 51-56. 
[5] N. N. Bogolyubov, "About One New Form of an Adiabatic Perturbation Theory in a Problem about Interaction Particles with a Quantum Field," Ukrainskii Matematicheskii Zhurnal (Russian Edtion), Vol. 2, 1950, pp. 3-24.

[6] V. K. Mukhomorov, "On a Spectra of the Coupled States of Polaron in a Limit of Adiabatic and Strong Coupling," Optics and Spectroscopy (USSR), Vol. 71, 1991, pp. 958-965.

[7] V. K. Mukhomorov, "Singlet and Triplet States of a Continuum Bipolaron for Adiabatic and Strong Coupling Limit," Optics and Spectroscopy (USSR), Vol. 74, 1993, pp. 544-655.

[8] V. K. Mukhomorov, "Bipolaron States of Electrons and Magnetic Properties of Metal-Ammonia Systems," Physica Status Solidi B, Vol. 219, 2000, pp. 71-89.

[9] V. K. Mukhomorov, "Ground and Excited States of a Three-Dimensional Continual Bipolaron," Physica Status Solidi B, Vol. 231, 2002, pp. 462-476.

[10] J. Jortner, "Energy Levels of Bound Electrons in Liquid Ammonia," Journal of Chemical Physics, Vol. 30, 1959, pp. 839-846.

[11] J. C. Thompson, "Electrons in Liquid Ammonia," Claredon, Oxford, 1976.

[12] N. F. Mott, "Metal-Insulator Transitions," Taylor and Francis, London, 1974.
[13] V. K. Mukhomorov, "Stability of Bipolarons, Electron-Electron Correlations, the Variational Principle, and the Virial Theorem," Physics of the Solid State, Vol. 48, 2006, pp. 864-870.

[14] V. K. Mukhomorov, "About the Problem of the Existence of a Three-Dimensional Bipolaron," Physica Scripta, Vol. 69, 2004, pp. 139-145.

[15] V. K. Mukhomorov, "Bipolaron Formations and Interpolaron Interactions in Dielectric Layers," Physica Scripta, Vol. 79, No. 6, 2009, pp. 065704-065709.

[16] I. Z. Fisher, "Statistical Theory of Liquids," University of Chicago Press, Chicago, 1965.

[17] A. I. Gubanov, "Quantum Electron Theory of Amorphous Conductors," Consultants Bureau, New York, 1965.

[18] E. J. Hart and M. Anbar, "The Hydrated Electron,” Wiley, New York, 1970.

[19] R. C. Dauthit and J. L. Dye, "Absorption Spectra of Sodium and Potassium in Liquid Ammonia," Journal of the American Chemical Society, Vol. 82, 1960, pp. 44724478.

[20] S. Arai and M. G. Sayer, “Absorption Spectra of the Solvated Electron in Polar Liquids: Dependence on Temperature and Composition of Mixture," Journal of Chemical Physics, Vol. 44, 1966, pp. 2297-2301. 